\title{
Major Accelerator Facilities for Nuclear Physics in Asia Pacific
}

\author{
Kazuhiro Tanaka \\ (kazuhiro.tanaka@kek.jp), \\ Chair of Asian Nuclear Physics Association (ANPhA). \\ Institute of Particle and Nuclear Studies (IPNS) and \\ Particle and Nuclear Physics Division, J-PARC Centre, \\ High Energy Accelerator Research Organization (KEK), \\ Oho 1-1, Tsukuba-shi, Ibaraki-ken, 305-0801 JAPAN.
}

\begin{abstract}
Asian Nuclear Physics Association (ANPhA) is the central organization representing nuclear physics in Asia Pacific. ANPhA is now preparing a list of accelerator facilities applicable for nuclear physics experiments in Asia Pacific. Among them, characteristics of the world class "Major" accelerator facilities were briefly summarized in Varenna2018 in comparing to similar facilities in Europe and North America.
\end{abstract}

\section{Introduction to ANPhA}

Asian Nuclear Physics Association (ANPhA) [1] was established in 2009 in Beijing, where representatives of the first four member countries of ANPhA gathered together in a founding meeting. $\mathrm{ANPhA}$ is the central organization representing nuclear physics in Asia Pacific and consist of eleven member countries and regions, i.e. Australia, China, Hong Kong, India, Japan, Kazakhstan, Korea, Mongolia, Myanmar, Taiwan, and Vietnam.

The basic objectives of ANPhA are;

1. To strengthen "Collaboration" among Asian nuclear research scientists through the promotion of nuclear physics and its transdisciplinary and applications,

2. To promote "Education" in Asian nuclear science through mutual exchange and coordination,

3. To "coordinate" among Asian nuclear scientists by actively utilizing existing research facilities,

4. To "discuss future planning" of nuclear science facilities and instrumentation in Asia.

In 2015, ANPhA decided to play a role as the Division of Nuclear Physics (DNP) of Association of Asia Pacific Physics Societies (AAPPS). AAPPS approved our proposal in 2016, and AAPPS-DNP was established. Now ANPhA chair is the chair of AAPPS-DNP. Practically, ANPhA (=AAPPSDNP) is an organization to discuss and pursuit issues in Asian nuclear physics community at present.

Participating countries or regions in ANPhA will appoint several (1 to 4) Board members for ANPhA. Board members elect one chairperson and several vice chairpersons by mutual election. The chairperson will also appoint a secretary from Board members. The Chairperson, Vice Chairperson, and Secretary constitute an Executive Officer team, and handle daily affairs.

ANPhA Board members meet together once a year at some appropriate place in one of ANPhA member countries or regions and exchange information of the status of nuclear physics in each country/region and have discussions on our future collaborations. This kind of meeting is organized in conjugation with ANPhA symposium on "Status of Nuclear Physics in Asia Pacific". The most recent (13th) ANPhA Board meeting was held in Beijing, China in September 14th in 2018. Next Board Meeting will be held in Korea in the fall in 2019. 
As the chair of AAPPS-DNP, ANPhA chair attends the extended Council meeting of AAPPS.

Another important activity of ANPhA is to organizing DNP-AAPPS (=ANPhA) awards for young Scientists [2] for ANPhA supported scientific meetings.

\section{ANPhA White Paper}

Nuclear physics is a typical accelerator-based science. However, in contrast to elementary particle physics, which is another field of science based on accelerators, nuclear physics requires to prepare a variety of accelerators to tackle the various problems involved. In other words, one needs a distributed approach and efforts, i.e. different accelerator types and energies, in order to find answers to the nuclear physics problems existing in our universe. The development of accelerator-based research facility always involves big construction work. It is also expensive and requires very large amount of money. Today we can understand that it is very difficult to prepare all kinds of accelerators necessary for the nuclear physics research in one country. Then it is becoming a common to advance research through international collaboration, i.e. international division of labor.

Even in Asia Pacific region, many advanced accelerator facilities have been constructed. Some of them are really world class facilities. ANPhA is now preparing a list of accelerator facilities applicable for nuclear physics experiments existing in Asia Pacific. The list is so called ANPhA White Paper [3]. This White Paper, the catalog of accelerators in Asia Pacific, is the most basic material for us to consider today's international collaboration within present accelerator facilities, and to establish the long range plan of the construction of accelerator facilities for our future activities of nuclear physics in Asia Pacific. Such international scheme of collaboration is originally global. Then the ANPhA White Paper can be important inputs for European and American colleagues of nuclear physics.

It should be noted that accelerator facilities originally prepared for nuclear physics research have many applications of science, such as material science, life science, medicine, and especially education and training of young students. Therefore ANPhA White Paper can be a good guide line for researchers in neighboring research field to expand their research to accelerator based science using near-by facilities.

Now 28 accelerator facilities for nuclear physics in Asia Pacific are listed in the ANPhA White Paper. Data will be updated frequently and the latest update was done in August 2018. Critical analysis of the present data will be made for future facility planning and for possible future international collaboration.

Data are now temporarily open on the KEK Indico system;

https://kds.kek.jp/indico/category/1706/

Notes for KEK Indico users, please find the username and password at the first page you opened (Most users) or "click for the password" on the page which you can find after closing the popup window to login (Google Chrome users).

\section{Major Accelerator Facilities in Asia Pacific}

Major facilities in Asia Pacific region are mainly locating in China (Heavy Ion Research Facility in Lanzhou (HIRFL), Beijing Tandem Accelerator National Laboratory (BTANL)), India (Variable Energy Cyclotron Centre (VECC)), Korea (RISP/RAON), and Japan (RIBF at RIKEN, J-PARC, and ELPH/LIPS). Most of them (HIRFL, BTANL, VECC, RISP/RAON and RIBF) are medium energy heavy-ion accelerator facilities and are competing to European and American Facilities such as SPIRAL2, HIE-ISOLDE and ARIEL-II. In addition, future extension plans of these Asian facilities are really aiming far beyond the wave front of the research of this field of nuclear physics. In this 
meaning, Asian research facilities are keeping world best positions in medium energy heavy-ion physics. Hadron physics facility in Asia Pacific (J-PARC) is also world leading facility. ELPH/LIPS facilities can provide world competitive photon beams for nuclear and hadron physics.

However, there are no high energy heavy-ion accelerators and colliders (such as ALICE in LHC in CERN, RHIC in BNL in USA, and NICA in DUBNA in Russia) in Asia Pacific region. In other word, we concentrated our research resources to medium energy heavy-ion physics and chosen to promote high energy heavy-ion physics at abroad (outside Asia). This strategy seems successful at present. However we have to check our strategy of this field of nuclear physics for our future research activities in Asia Pacific. For example, I'm wondering that too much concentration might be happening in medium energy heavy-ion accelerator facilities in Asia Pacific region. This concentration is happening also in Europe and America. Should we be much more careful on our investment for our future activities in nuclear physics, which should have much wider spectrum, I think?

Some of our "Major Facility status and future plans" are introduced in the following Chapters.

\section{$4 \quad$ Chinese Facilities}

Construction of accelerator facilities in China is in very much strategic and clever way. They constructed ordinal experimental facility based on the tandem electrostatic accelerator in 1986 in Beijing and construction of the experimental facility based on Split Sector Cyclotron (SSC) followed it in 1988 in Lanzhou.

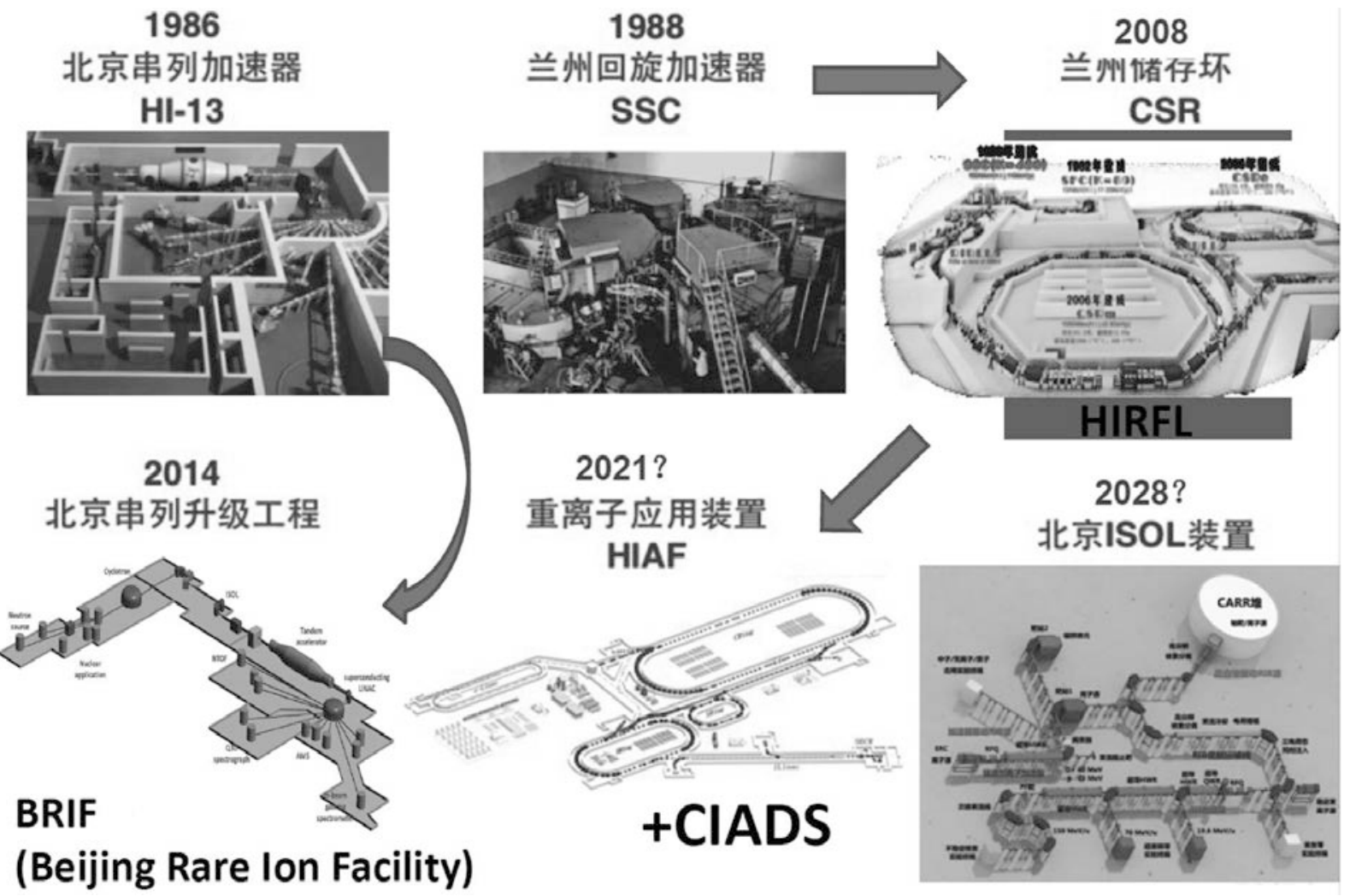

Fig. 1: Chinese accelerator facilities for nuclear physics.

After the successful operation of both facilities for approximately 20 years as "normal” beam facilities, 
accumulator rings were constructed in SSC facility in 2008 and SSC was used as the injector to rings. "Unstable" nuclear beams produced through projectile fragmentation from stable (normal) nuclear beams obtained from SSC were accumulated in rings and extracted for experiments after energy boosted and beam quality improved in the rings. For Beijing facility they added small cyclotron to produce "unstable" nuclear beams by using target ion source. Proton beam obtained from small cyclotron irradiated the target material which was heated up by beam power as well as electrical heater. Unstable nuclei produce in the target material through nuclear reactions were thus evaporated from the surface of the target material and collected for the re-acceleration by the tandem electrostatic accelerator. Then the tandem facility and SSC facility were well converted to the most modern "unstable” nuclear beam facilities.

Their next steps are the construction of very High Intensity Accelerator Facility (HIAF) for the production of unstable nuclear beams based on the projectile fragmentation, which is the natural extension from Lanzhou's SSC facility but constructed in the other place, i.e. Huizhou city, and the SUPER ISOL facility based on the combination of nuclear reactor and linear accelerator in Beijing, i.e. Beijing ISOL.

\section{$5 \quad$ Korean Facility}

The major accelerator facility under construction in Korea is RAON (Rare isotope Accelerator complex for ON-line experiments) of RISP (Rare Isotope Science Project) hosted by IBS (Institute of Basic Science). This is the first big scientific project in Korea concerning to the construction of the world class accelerator complex. Location of RAON is Sindong area in Daejeon city, which is almost the central part of South Korea and almost 2-3 hours travel by KTX fast train from both Seoul and/or Pusan. The ground breaking for accelerators and experimental buildings was done on Feb. 13th in 2017.

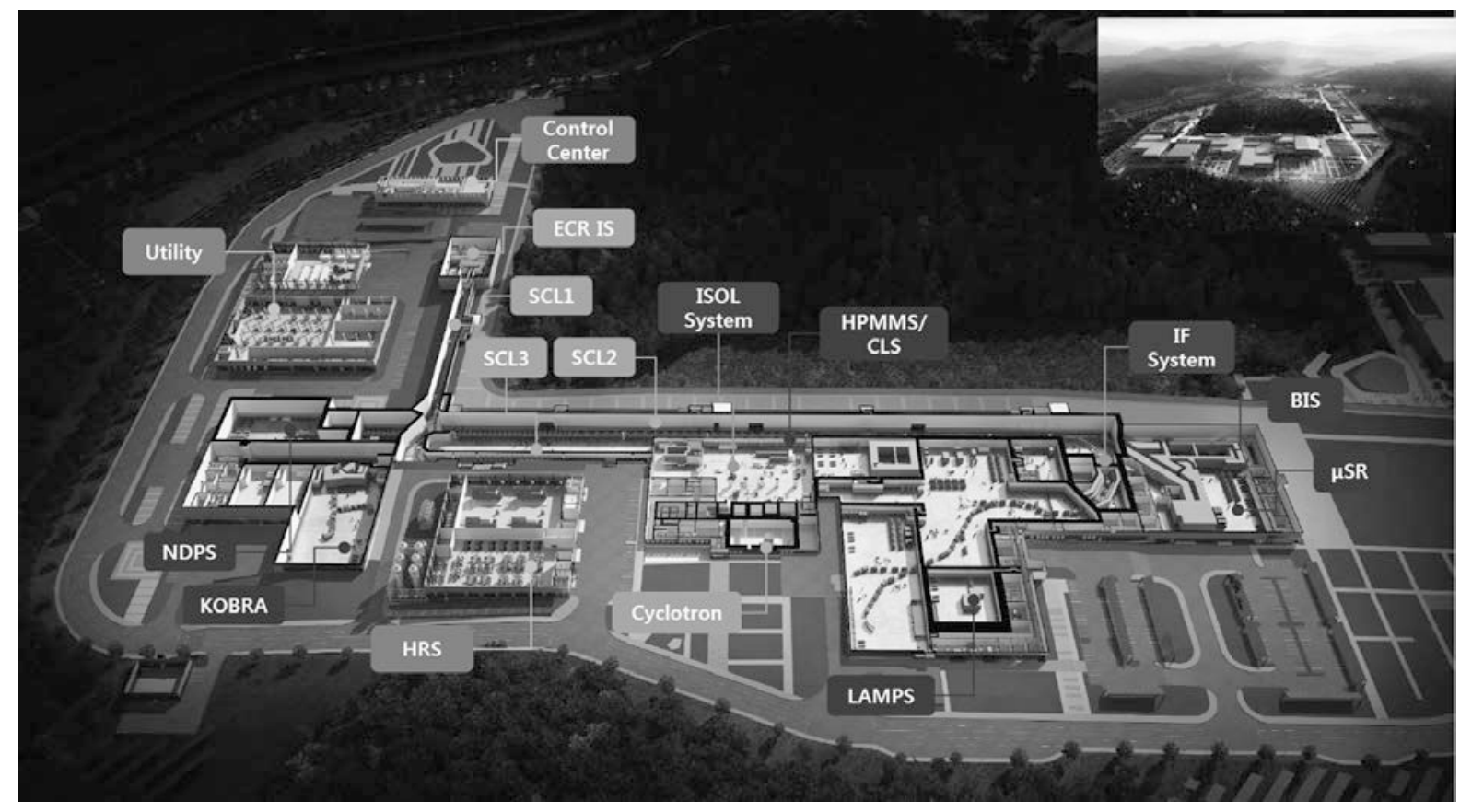

Fig. 2: RAON accelerator complex of RISP of IBS in Korea.

RAON accelerator consists of three superconducting linear accelerators. Combining three linacs, 
acceleration of normal heavy-ion beams and unstable nuclear beams extracted from target ion source to sufficiently high energies to projectile fragmentation. As results RAON can provide much higher intensity unstable nuclear beams for experiments than any other facilities in the world. For the target ion sources, high intensity proton cyclotrons are introduced as drivers.

$R \& D$ of superconducting accelerator devices have already started and test cryo-module of acceleration cavity showed sufficiently high field gradient with less heat load than expected, i.e ready for mass production. Operation test of ISOL target ion sources has started at hot-cell mockup. Remote maintenance scheme of the target ion source will be tested there.

\section{Japanese Facilities}

There are several large scale accelerators in Japan as shown in Figure 3. Among them following 3 research complexes were endorsed by Japanese Nuclear Physics Executive Committee in 2016 as main middle term ( $\sim 5$ years) important future plans of nuclear physics in Japan. These are;

- J-PARC (KEK)

$>$ Hadron/nuclear physics with hadron beams

$\diamond \quad$-> Hadron Hall extension.

$>$ Fundamental Physics and Particle physics with muons

$\diamond \quad$-> mu-e conversion (COMET), g-2.

- $\quad$ RIBF (RIKEN)

$>$ RIBF upgrade for intensity x30

$\diamond \quad->$ Expand neutron-rich heavy element productions to trans-uranium.

$\diamond \quad$-> Production of superheavy $\mathrm{z}=119$ element and beyond.

- ELPH (Tohoku Univ.) and LEPS@SPring-8 (RCNP, Osaka Univ.),

$>$ Hadron physics with $\mathrm{GeV}$ electron and photon beams

$\diamond \quad$-> Detector/Beam upgrades.

In addition to them, two research fields were selected as important subjects for Japanese nuclear physics;

- High energy heavy-ion collision (LHC, RHIC, J-PARC)

$>$ QGP properties, QCD phase diagram, High density nuclear matter. $\diamond \quad$-> ALICE upgrade, s-PHENIX/STAR upgrade, J-PARC-HI R\&D.

- Nuclear theory

> Hadrons via lattice QCD, nuclear structure via Monte Carlo shell model, etc.

$\diamond \quad 9$ projects with K-computer and beyond.

J-PARC in KEK and RIBF in RIKEN are main two-top facilities of Japanese nuclear physics community. Then extension of Hadron Experimental Hall and 30 times intensity upgrade of RIBF are two-main big future plans in Japan. 


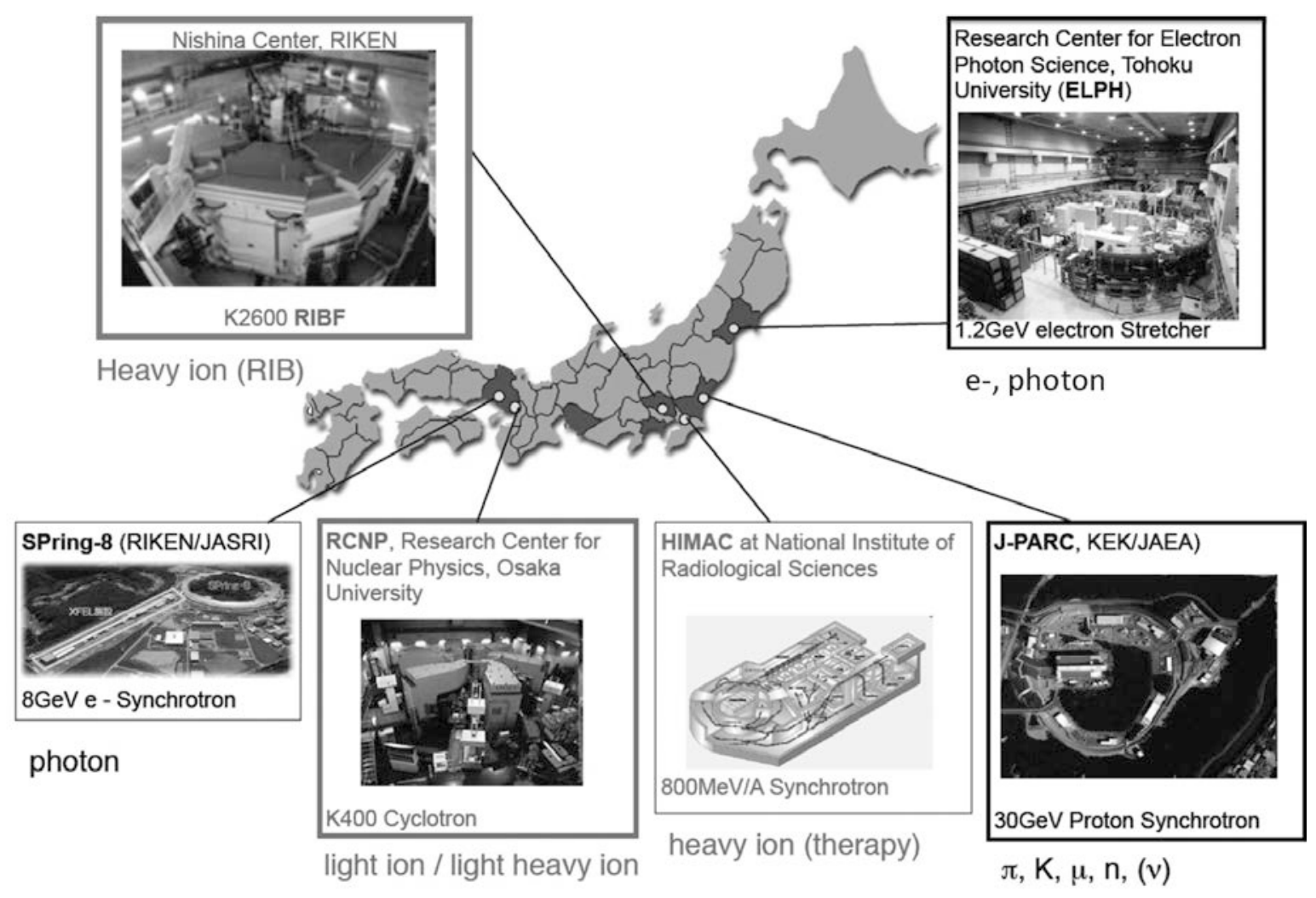

Fig. 3: Large scale accelerator complexes located in Japan.

Schematic layout of RIKEN-RIBF is shown in Figure 4. RIKEN-RIBF consists of several types of ring cyclotrons connected in cascade and one big superconducting ring cyclotron, SRC. Unstable nuclear beams are produced by projectile fragmentation (PF) and a large solid angle PF separator, the BigRIPS, is in operation. Upgrade for 30 times higher intensity is mainly performed by upgrading injector LINAC and by the modification of SRC and BigRIPS in order to accept higher intensity primary nuclear beams. High intensity unstable nuclear beam thus produced will be used for the search of new superheavy elements such as $Z=119,120$ and beyond. This upgrade project is named as "Landing to Stable Island".

J-PARC (Japan Proton Accelerator Research Complex) is the brand-new and the most advanced accelerator facility in Japan. J-PARC consists of three accelerators, i.e. $400 \mathrm{MeV}$ Linac, $3 \mathrm{GeV}$ Rapid Cycle Synchrotron (RCS) and 50 GeV-PS (Main Ring, MR). The bird eye view of J-PARC is shown in Fig. 5. The most important characteristic of J-PARC is its high design beam power, which is $1 \mathrm{MW}$ for RCS and $0.75 \mathrm{MW}$ for MR. RCS provides its intense proton beam to neutron spallation source (n) and pulsed muon source $(\mu)$ prepared in Materials and Life Science Facility (MLSF). Some fraction of the beam extracted from RCS is injected to MR and accelerated up to $30 \mathrm{GeV}$. Two extractions from MR were constructed. One is the fast extraction for Neutrino Beam Facility $(v)$ for long baseline neutrino oscillation experiment, $\mathrm{T} 2 \mathrm{~K}$, and the other is the slow extraction for counter experiments in Hadron Experimental Facility (Hd). Four experimental facilities (n, $\mu, v$ and $\mathrm{Hd}$ ) could provide their characteristic intense secondary beams for experimental users.

The highest proton beam energy of MR is now only $30 \mathrm{GeV}$ instead of its design energy of $50 \mathrm{GeV}$. It is mainly because of the budget problem for preparing power supplies of MR magnets. 
RIBF - a new generation RIB facility in operation with world highest capability of providing RI beams

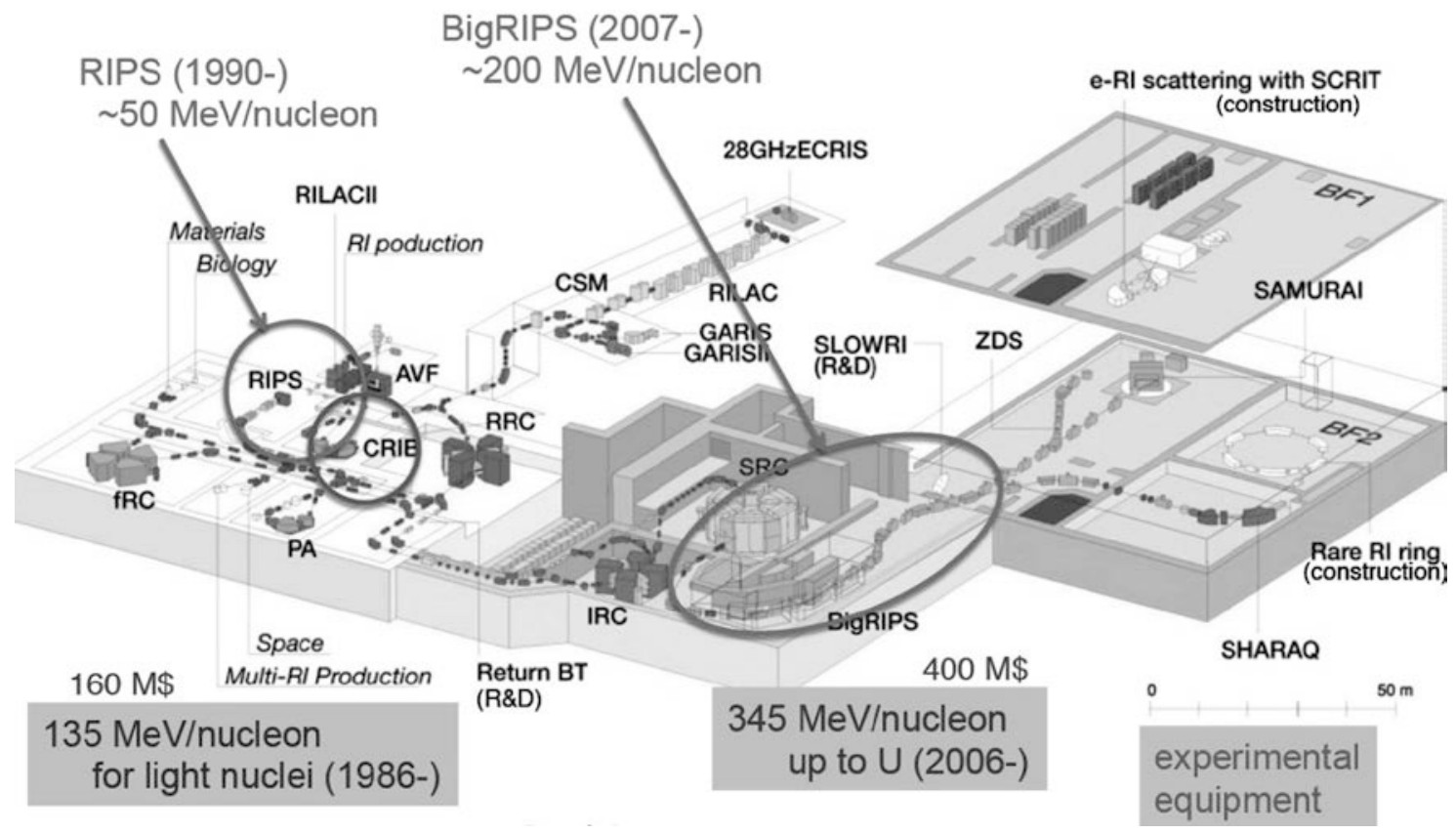

Fig. 4: Schematic layout of RIBF-RIKEN accelerator complex.

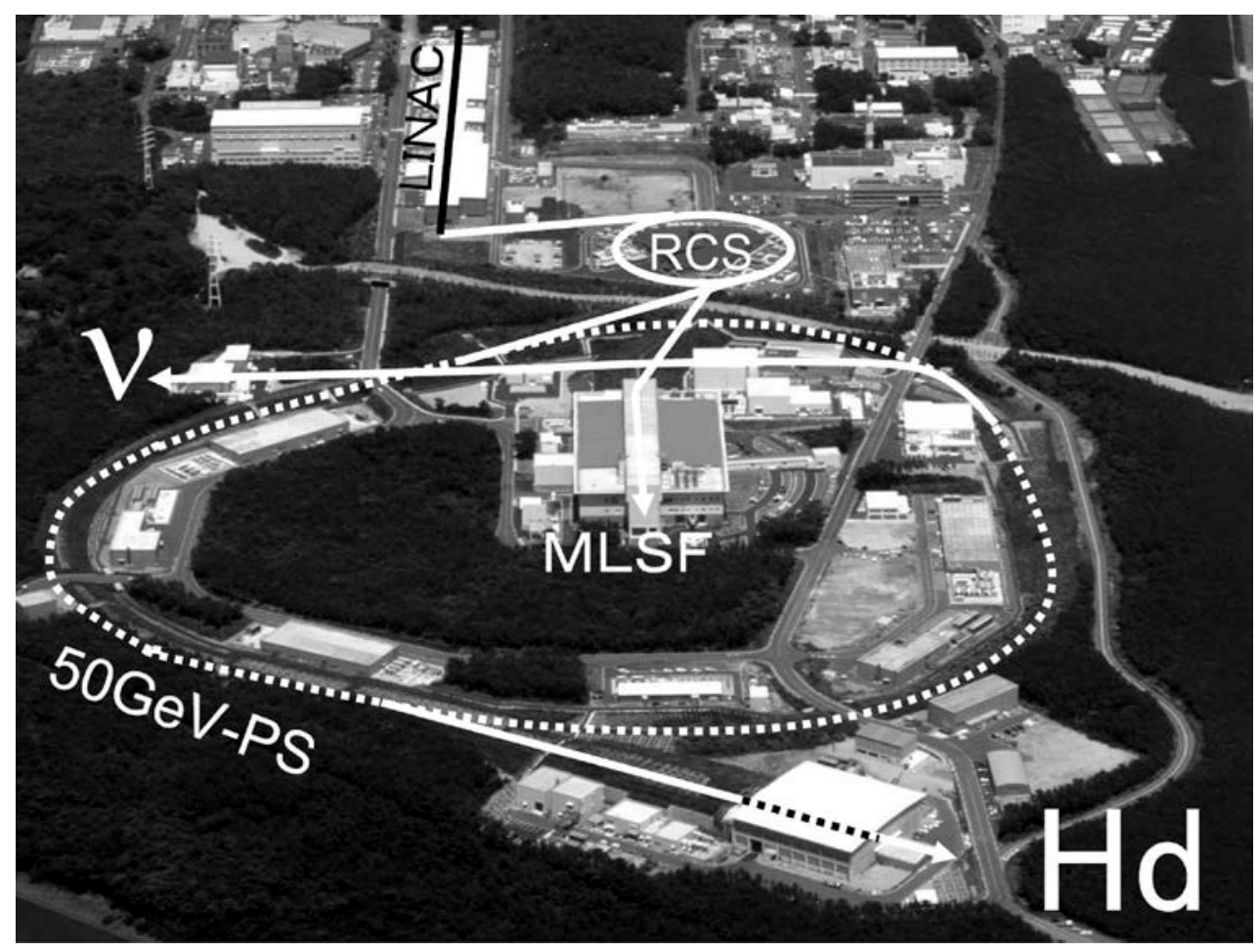

Fig. 5: J-PARC site at Tokai campus of JAEA. 
Major future project of J-PARC for nuclear physics is the extension of the Hadron Hall three times as shown in Figure 6.

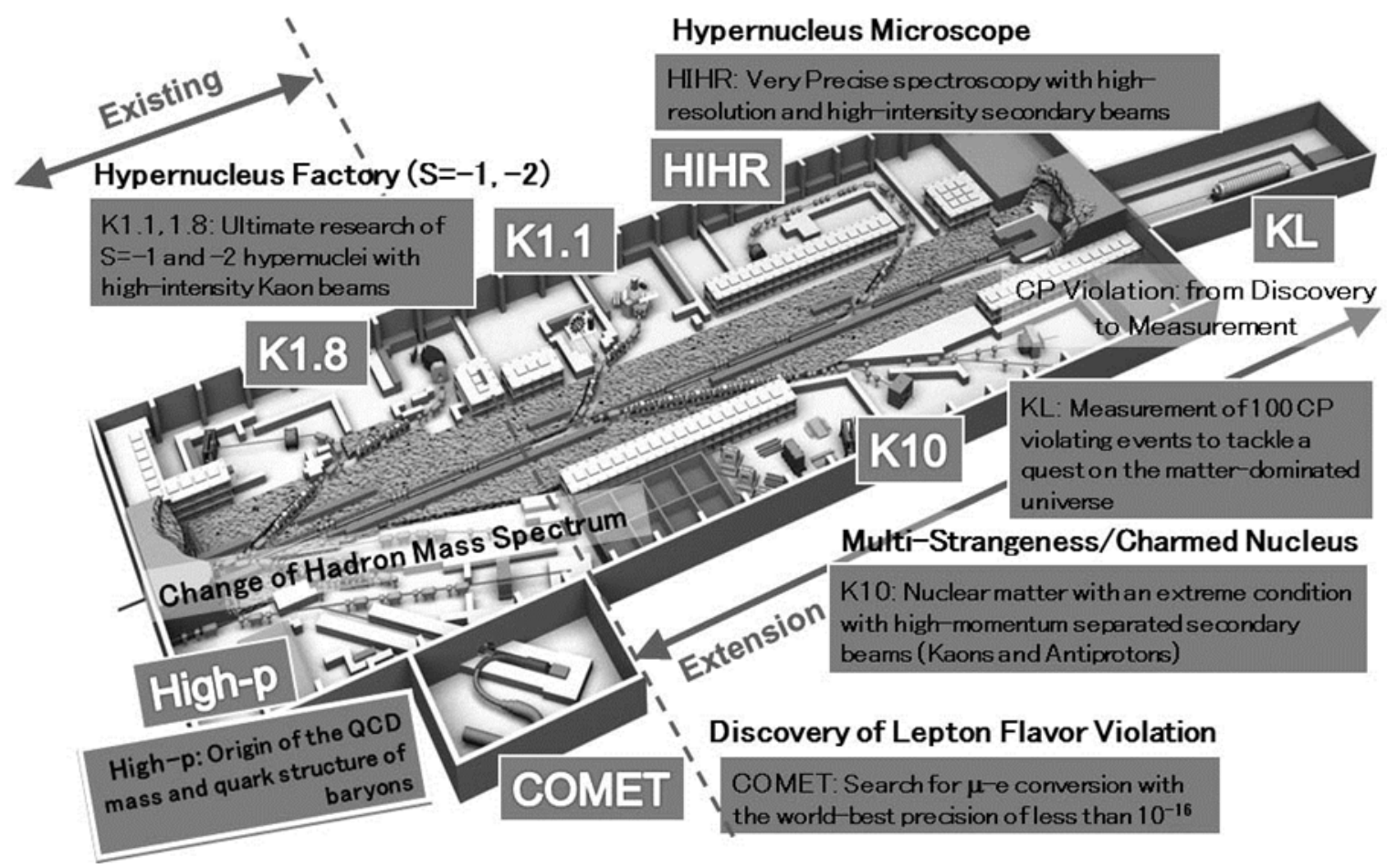

Fig. 6: Drawing of the extension of Hadron Experimental hall. Two new target stations are constructed and four new secondary beam lines are connected to these two targets. Very forward angle neutral kaon beam line, KL, high momentum separated kaon/antiproton beam line, K10, very high resolution dispersion matching beam line, HIHR, and very low energy separated kaon beam line, K1.1, are now under consideration. High-p beam line with COMET branch is now under construction at present Hadron Experimental hall.

\section{Acknowledgement}

The author would like to express his sincere thanks to Board members of Asian Nuclear Physics Association (ANPhA), who prepared the data for ANPhA White Paper.

\section{References}

[1] ANPhA: http://ribf.riken.jp/ANPhA/

[2] Kazuhiro Tanaka, "The First Year of the ANPhA (AAPPS-DNP) Awards for Young Scientists", AAPPS Bulletin, Vol. 28, No. 1, pp. 43-45.

[3] ANPhA White Paper: https://kds.kek.jp/indico/category/1706/

Notes for KEK Indico users, please find the username and password at the first page you opened (Most users) or "click for the password" on the page which you can find after closing the popup window to login (Google Chrome users). 